\title{
Quantifying and testing coexistence mechanisms arising from recruitment fluctuations
}

\author{
Peter Chesson* \\ Section of Evolution and Ecology, The University of California, Davis, CA 95616-5270, USA
}

Received 21 April 2003

\begin{abstract}
Temporal fluctuations in recruitment are involved in two distinct coexistence mechanisms, the storage effect and relative nonlinearity of competition, which may act simultaneously to stabilize species coexistence. It is shown that comparisons of recruitment variation between species at high versus low densities can test whether these mechanisms are responsible for stable coexistence. Moreover, under certain circumstances, these comparisons can measure the total coexistence stabilizing effect of the mechanism. These comparisons are clearest for the situation of an invader (a species perturbed to low density) in the presence of its competitors, termed residents. Then average invader-resident differences in the variances of log recruitment, potentially weighted by adult survival rates and species' sensitivities to competition, are proportional to the overall stabilizing effect of the storage effect and relative nonlinearity of competition. Less effective comparisons are available for species naturally at high and low densities or with substantial mean differences in average fitness. These developments lead also to a technique of partitioning the long-term lowdensity growth rate of a species into community average measures of stabilizing mechanisms, deviations from these measures, and other factors. The community average measure is argued as most appropriate for understanding the ability of a coexistence mechanism to stabilize coexistence. Individual species' deviations from the community average indicate the ways in a which a coexistence mechanism may affect average fitness differences between species either enhancing or diminishing the ability of a given set of species to coexist, depending on other factors. This approach provides a general new tool for analyzing species coexistence. (C) 2003 Elsevier Inc. All rights reserved.
\end{abstract}

Keywords: Recruitment variation; Storage effect; Relative nonlinearity; Coexistence

\section{Introduction}

According to one classification, there are two distinct theoretical mechanisms of coexistence that arise from temporal fluctuations in the environment (Chesson, 1994; Chesson and Huntly, 1997). The stronger of these, termed "the storage effect," is a formalization of the concept of temporal environmental niche differentiation (Chesson et al., 2001). The other mechanism, "relative nonlinearity of competition" has seemed restricted in its ability to explain the coexistence of more than a few species in a robust way (Chesson, 1994; Abrams and Holt, 2002), although recent work on nonlinear multi-resource models is suggestive of an important role also for this mechanism (Huisman and Weissing, 2002). Moreover, the results here show that

\footnotetext{
${ }^{*}$ Fax: + 530-752-1449.

E-mail address: PLChesson@UCDavis.edu.
}

even though relative nonlinearity of competition may have difficulty explaining much diversity when operating alone, it may modify the outcomes of other mechanisms determining their overall ability to maintain diversity in any community. While much is understood about these mechanisms theoretically, their operation in nature is poorly understood. One reason for this situation is the difficulty of understanding how these mechanisms can be tested in nature.

The purpose of this article is two-fold. First, it aims to help redress the difficulty of testing coexistence mechanisms based on recruitment variation by developing theoretical results that help justify and expand tests of the storage-effect coexistence mechanism, including tests of the storage effect in combination with relative nonlinearity of competition. Second, the problem of testing models based on recruitment variation raises the important issue of how the strengths of coexistence mechanisms can be assessed. In previous 
work (Chesson, 1994, 2000a), it was possible to quantify the effects of particular coexistence mechanisms on the persistence of individual species. However, coexistence is a community property, and if a coexistence mechanism is to be quantified, it seems most appropriate to do so at the community level. Thus, the second aim of this article, which is needed to fully achieve the first, is to solve the problem of quantifying the strengths of coexistence mechanisms at the community level. Although solving this problem was motivated by the question of testing mechanisms based on recruitment variation, it has broad applications to the theoretical and empirical analysis of species coexistence.

\subsection{Past approaches to testing the storage effect}

There are several approaches to testing the storage effect based on patterns of recruitment fluctuations over time. Warner and Chesson (1985) developed a method for partitioning long-term population growth rates into components due to recruitment fluctuations and components due to other factors, and this technique was applied most notably to testing the storage effect for coexisting Daphnia species (Caceres, 1997). Although this technique reveals the effects of recruitment fluctuations on the dynamics of an individual species, it does not directly address interactions between species. However, Chesson and Huntly (1989) pointed out that when the storage effect is operating, population growth rates might tend to fluctuate more for a species at low density than for a species at high density. If these growth-rate fluctuations are driven by recruitment fluctuations, then higher recruitment fluctuations should be seen for a species at lower density. Recently, Kelly and Bowler (2002) advanced this idea as a test of the storage effect in forest trees. They based their predictions on a particular version of the lottery model (Chesson and Warner, 1981) where only one species is sensitive to the environment, with the environment fluctuating between two states only. However, no systematic exploration of the use of patterns of recruitment fluctuations to test the storage effect has been done. Here that gap is filled.

\subsection{Overview of recruitment fluctuations and the storage effect}

Recruitment fluctuations are features of many species in many different systems. For example, reef fish species often have highly variable numbers of larvae arriving at settling sites where they enter the juvenile age class (Doherty and Fowler, 1994; Dixon et al., 1999). In plant species, seed production, and seed germination can be highly variable (Pake and Venable, 1995, 1996). In addition, recruitment variation can be interpreted more broadly to include recruitment of leaves on individuals in a population, or recruitment of biomass or resources, each of which in some circumstances can be highly temporally variable (Warner and Chesson, 1985; Chesson et al., 2001).

Variable recruitment at one stage in the life cycle often leads to variable recruitment at later stages. For example, variable recruitment of juvenile fishes may lead to variable recruitment of adult fishes (Abrams, 1984); and strongly spatially and temporally varying conditions for seed production may lead to variable recruitment of seedlings, potentially flowing down a chain to variable recruitment of saplings and mature trees. In these and many other cases, relatively brief intermediate stages feed variable input to a longer-lived stage. Indeed, when variable recruitment occurs, there is often some relatively persistent stage in the life cycle that has the effect of buffering recruitment variation. For example, in annual plants, a persistent seedbank often accompanies highly variable recruitment of seeds into it (Pake and Venable, 1996). In trees, both seedlings and adult trees may show high levels of persistence. Typically, at any given time, a persistent stage consists of individuals from several to many past recruitment events. The number of individuals in a persistent stage is thus a discounted sum of recruitment over time, with distant recruitment events discounted according to the rate at which individuals leave the stage by death or entrance of another stage (Warner and Chesson, 1985).

The presence of a stage in the life cycle that buffers recruitment variation does not necessarily mean that recruitment variation is unimportant. Indeed, the storage-effect coexistence mechanism relies on such buffering effects of persistent stages, because these prevent catastrophic population decline when poor recruitment occurs. As a consequence, a population benefits from favorable recruitment events without the gains during favorable times being canceled by population declines during periods of poor recruitment (Chesson, 1994). These issues are most important for recovery of a species from low density, stabilizing its presence in the system, because, at low density, environmental factors that might favor high recruitment rates are not opposed by intraspecific competition.

These ideas are understood in the theory of the storage effect as follows: First, density-independent environmental factors have a direct impact on per capita recruitment. For example, in coral reef fishes, survival rates of larval fish prior to settlement may be affected by environmental factors in the water column (Dixon et al., 1999). For annual plants, environmental factors can have large effects on germination rates (Baskin et al., 1993) and growth rates of seedlings (Pake and Venable, 1995). Second, competition, both within and between species, reduces the actual recruitment that occurs (Chesson and Huntly, 1989). Indeed, recruitment variation reflects high sensitivity of the recruitment process to both environmental and competitive influences 
(Chesson, 1990). Third, direct environmental effects on recruitment alter the competition that occurs during recruitment by altering the density of individuals that are competing for the resources needed for recruitment. For example, if more tree seedlings appear in the forest due to environmental effects, competition between seedlings can be expected to be greater, and thus recruitment into the adult stage will be affected both by the direct environmental effect producing the larger number of seedlings, and the greater level of competition that they experience. In this situation, the environment has two different effects on recruitment. The first of these is its effect on the production of seedlings (a direct effect of the environment), and the second is its effect on the total magnitude of competition between these seedlings (the indirect effect of the environment). Thus, the direct effects of environment on recruitment, and the effects of competition on recruitment, can be expected to be correlated, i.e. can be expected to covary. This phenomenon is called covariance between environment and competition (Chesson, 1994). The precise definition and measurement of covariance between environment and competition are given below.

For any given variance in the direct effect of the environment and the effect of competition, recruitment variation decreases with the magnitude of covariance between environment and competition. Because species differ in their patterns of direct response to the environment over time, covariance between environment and competition is greater for a species experiencing mostly intraspecific competition versus a species experiencing mostly interspecific competition (Chesson, 1994). A species at high density would be expected to have more intraspecific and less interspecific competition than a species at low density. Thus, a species at high density would have greater covariance between environment and competition, and thus weaker recruitment fluctuations than a species at low density. According to the theory of the storage effect (Chesson, 2000b), in the presence of a long-lived life-history stage that buffers variation, this difference in covariance between environment and competition for high- versus low-density species gives an average population growth rate advantage to a species at low density, promoting its coexistence with its competitors. Indeed, together with a persistent stage in the life cycle, this covariance difference is the heart of the storage-effect coexistence mechanism. Because of the likely association of covariance differences with recruitment-fluctuation differences, we ask here to what extent observations on recruitment-fluctuation differences can be used to test the storage-effect coexistence mechanism.

In pursuing this goal we consider two different ways of viewing low density. In the first case, a species at low density is there only temporarily. It may have been experimentally reduced to low density, have just arrived in the system, or have experienced a sequence of unfavorable environmental events that have brought it to very low density, from which it will recover. In these situations, the species is referred to as an invader, corresponding to the use of invader in theoretical invasibility coexistence analysis (Turelli, 1978; Ellner, 1989; Chesson, 1994). In the second case, species are distinguished by their long-term mean densities. A lowdensity species is therefore low on average.

\section{Models and mechanisms}

For perennial organisms, a general model in which recruitment fluctuations promote coexistence by the storage effect takes the form

$N_{j}(t+1)=\left(1-d_{j}\right) N_{j}(t)+R_{j}(t) N_{j}(t)$,

where $N_{j}(t)$ is the density of adults of species $j$ at time $t$, $d_{j}$ is the fraction of adults dying during one unit of time and $R_{j}(t)$ is the per capita number of new recruits to species $j$ in the time interval $t$ to $t+1$ (Chesson, 1994). Eq. (1) might be generalized to consider age-structured mortality and fecundity, but results of Dewi and Chesson (2003) suggest that such age structure would not have major effects on the results discussed here. Per capita recruitment, $R_{j}(t)$, is assumed to reflect the direct response of the organisms to the environment (the environmental response, $E_{j}(t)$ ), and a response to competition, $C_{j}(t)$. The recruitment rate might then reasonably satisfy the formula

$R_{j}(t)=e^{E_{j}(t)-C_{j}(t)}=e^{E_{j}(t)} / e^{C_{j}(t)}$

or equivalently

$\ln R_{j}(t)=E_{j}(t)-C_{j}(t)$,

which defines recruitment as additive in the environmental and competitive responses on a log scale, a scale that proves useful in both data and theoretical analysis (Chesson, 1982). The most important feature of this model, however, is that recruitment is represented as a function of a direct environmental effect, $\exp \left(E_{j}(t)\right)$, divided by a function of competition, $\exp \left(C_{j}(t)\right)$. Indeed, in cases where competition can be removed by reducing densities, $E_{j}(t)$ and $C_{j}(t)$ can be operationally defined by the formulae

$E_{j}(t)=\ln R_{j}^{0}(t)$,

$C_{j}(t)=\ln \left(R_{j}^{0}(t) / R_{j}(t)\right)$,

where $R_{j}^{0}(t)$ is the recruitment rate in the absence of competition, and $C_{j}(t)$ is then the reduction in $\ln$ recruitment due to the action of competition. These definitions parallel empirical approaches to determining the intensity of competition (Grace, 1995). 
A plausible model for $C_{j}(t)$ is

$C_{j}(t)=\sum_{l=1}^{n} a_{l} e^{E_{l}(t)} N_{l}(t)$

where the term $\exp \left(E_{l}(t)\right) N_{l}(t)$ simply represents the number of juveniles of species $l$ that are competing with each other and with other species, and $a_{l}$ is the competitive effect of a juvenile of species $l$. In this expression, $\exp \left(E_{l}(t)\right)$ represents the product of the per capita fecundity of species $l$ and the juvenile survival rate in the absence of competition, and would therefore be the recruitment rate in the absence of competition. Thus, this model assumes that the principle competition is between juveniles, presumably for resources that they need to mature.

An important alternative form of $C_{j}(t)$ with similar properties to form (6) is the lottery form (Chesson, 1994), where

$C_{j}(t)=\ln \left\{\frac{\sum_{l} e^{E_{l}(t)} N_{l}(t)}{\sum_{l} d_{l} N_{l}(t)}\right\}$,

which is simply the natural $\log$ of the ratio of the number of juveniles competing for settling sites to the number of settling sites that are available due to death of adults. The general results discussed here, however, do not depend on the details of the formula for $C_{j}(t)$, and apply equally to the lottery model, the model given by (6), and similar models including the generalization of Eqs. (6) and (7) given in the appendix as Eq. (A.5). The defining features of the major part of our development are Eqs. (1) and (2), with the additional assumption that $C_{j}(t)$ does not depend on $j$, i.e. all species experience the same magnitude of competition. A more general situation in which the $C_{j}(t)$ are proportional between species is considered also, but in less detail. For both situations (equal or proportional $C_{j}(t)$ ), equilibrial coexistence is precluded, i.e. in the absence of fluctuations in recruitment, the species could not coexist. Mechanisms of stable coexistence are then restricted to the storage effect and relative nonlinearity of competition (Chesson, 1994), which we wish to assess by measuring variation in recruitment.

Of interest is relative recruitment variation, not the variance of recruitment, and relative recruitment variation is appropriately measured as the variance of $\ln R_{j}(t)$, because the $\log$ scale is a relative scale. Alternatively, the squared coefficient of variation may be used. These measures give numerically similar values, and behave in similar ways provided the variance is not too large. The variance of $\ln R_{j}(t)$ is much simpler for theoretical investigations, but the squared coefficient of variation may be preferred in empirical studies when some values of $R_{j}(t)$ might be zero.
Using expression (3) for $\ln R_{j}(t)$ we see that

$$
V\left(\ln R_{j}(t)\right)=V\left(E_{j}(t)\right)+V\left(C_{j}(t)\right)-2 \operatorname{Cov}\left[E_{j}(t), C_{j}(t)\right]
$$

and hence that recruitment variation $\left(V\left(\ln R_{j}(t)\right)\right.$, and covariance between environment and competition $\left(\operatorname{Cov}\left[E_{j}(t), C_{j}(t)\right]\right)$, are indeed negatively related to one another. As discussed above, the storage-effect coexistence mechanism relies on changes in $\operatorname{Cov}\left[E_{j}(t), C_{j}(t)\right]$ between high and low density. Does this mean that the operation of the mechanism, and a general test of the mechanism, is possible by examining variation in recruitment at different densities, rather than directly measuring $\operatorname{Cov}\left[E_{j}(t), C_{j}(t)\right]$ ?

\subsection{Conditions for coexistence}

The model in the section above is a special case of a general model analyzed by Chesson (1994) in which the per capita growth rate, defined as $r_{j}(t)=\ln N_{j}(t+1)$ $-\ln N_{j}(t)$, takes the form

$r_{j}(t)=g_{j}\left(E_{j}(t), C_{j}(t)\right)$

for some function $g_{j}$. For the recruitment variation model given above

$g_{j}\left(E_{j}, C\right)=\ln \left\{1-d_{j}+e^{E_{j}-C}\right\}$,

where $t$ is suppressed for simplicity of notation, and $C$ is not given a subscript because we shall assume that it is the same for all species, as discussed above. To determine species coexistence in a nonequilibrium fluctuating environment scenario, one uses the invasibility criterion (Turelli, 1981; Chesson, 1994), and evaluates the long-term growth rate of each species at low density. If this long-term growth rate is positive for each species, they coexist by the invasibility criterion. Note that recovery from low density is a definition of stable coexistence, and is to be contrasted with neutral coexistence applicable to neutral models (Chesson and Huntly, 1997; Hubbell, 2001). The long-term lowdensity growth rate is denoted $\bar{r}_{i}$, and is evaluated as the expected value of $r_{i}(t)$ for species $i$ considered as an invader, i.e. with species $i$ set to zero and the other species (termed residents) having the joint stationary distribution that they achieve in the absence of species $i$.

Under the assumption that the $E_{j}(t)$ undergo fluctuations of small magnitude, it is shown in Chesson (1994) that $\bar{r}_{i}$ can be expressed in the form

$\bar{r}_{i} \approx \bar{r}_{i}^{\prime}-\Delta N_{i}+\Delta I_{i}$,

where $\bar{r}_{i}^{\prime}$ is a contribution due to equilibrium mechanisms of coexistence, $\Delta N_{i}$ measures the effects of relative nonlinearity, $\Delta I_{i}$ measures the storage effect, and the approximation error is small relative to the variance, $V\left(E_{j}\right)$, of $E_{j}(t)$, as discussed in the appendix. Results of Chesson (1994) are adapted in the appendix to give the 
Table 1

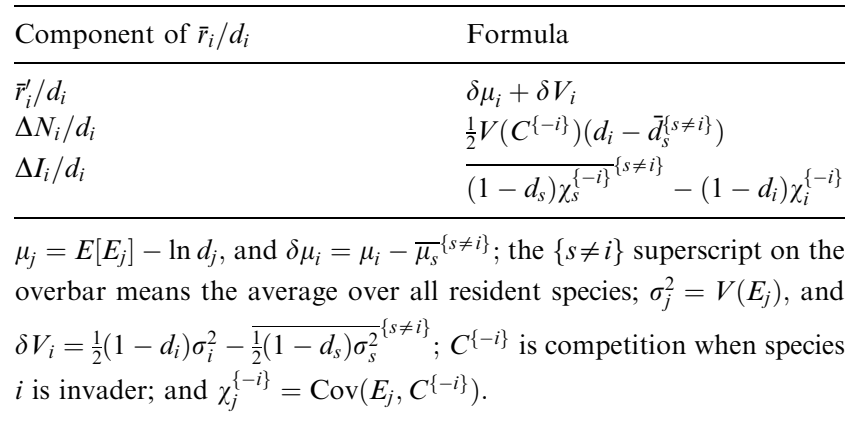

formulae in Table 1 for the various terms in Eq. (11). Note that each term in Table 1 has been rescaled by the common factor $d_{i}$. This rescaling amounts to a change of units from an absolute time scale, for example years, to a per generation scale for each species, because $1 / d_{i}$ is the generation time for species $i$. In effect, it converts these quantities to natural timescales, and leads to much more sensible results in the material that follows.

The storage effect $\left(\Delta I_{i} / d_{i}\right)$ is represented in Table 1 as a comparison of invader $(i)$ and resident $(s)$ covariance between environment and competition, multiplied by adult survivorship. As discussed above, when the species have different responses to the environment, covariance between environment and competition for invaders is expected to be lower than covariance between environment and competition for residents, tending to make $\Delta I_{i}$ positive for all species, and promoting their persistence in the system. By contrast, the first line of the table $\left(\bar{r}_{i}^{\prime} / d_{i}\right)$ sums to zero over species. Thus, unless this term is zero for all species, it will be negative for some species and positive for others. This term is fundamentally a comparison of resident and invader growth rates at a fixed average level of competition (see the appendix), and leads to a strict ranking of species for the given environmental conditions.

If the storage effect $\left(\Delta I_{i} / d_{i}\right)$ is large enough, it can cancel the negative values of $\bar{r}_{i}^{\prime} / d_{i}$ that some species have yielding positive low-density growth rates $\left(\bar{r}_{i}\right)$ for all species, permitting species coexistence. To see how this happens, it is instructive to consider the special case in which the species have equal adult death rates $\left(d_{j}\right)$, and the environmental response fluctuations have the symmetric variance and covariance structure: $V\left(E_{i}\right)=\sigma^{2}$ and $\operatorname{Cov}\left(E_{i}, E_{j}\right)=\rho \sigma^{2}$, for all species pairs, $i \neq j$, with the vector of environmental responses, $\left(E_{1}(t), E_{2}(t), \ldots, E_{n}(t)\right)$, being independent and identically distributed over time. Then, the storage effect reduces to the especially simple form

$\frac{\Delta I}{d_{i}}=\frac{\sigma^{2}(1-\rho)(1-d) B}{n-1}$,

where the positive constant $B$ depends on the particular form of competition as discussed in the appendix. Note that the storage effect is here simply proportional to the species-specific component, $\sigma^{2}(1-\rho)$, of the variance in a species' environmental response, $E_{i}(t)$. In this situation also, $\Delta N$ is zero, $\bar{r}_{i}^{\prime} / d_{i}$ reduces to $\delta \mu_{i}$, and so

$\frac{\bar{r}_{i}}{d_{i}}=\delta \mu_{i}+\frac{\sigma^{2}(1-\rho)(1-d) B}{n-1}$.

The species coexist if this quantity is positive for every species. This condition also has a simple meaning. The term $\delta \mu_{i}$ can be considered to be the average fitness advantage that a species has over its competitors in the system. The average of this value over species is zero, and therefore some species have negative fitness advantages, i.e. are disadvantaged in average fitness. These species would be competitively excluded if it were not for the second term, $\sigma^{2}(1-\rho)(1-d) B /(n-1)$, which is positive, and independent of the first term. Therefore, a large enough value of the second term overcomes all fitness disadvantages and allows all species to have positive values of $\bar{r}_{i}$, and hence coexist.

If the adult death rates are not equal, the relative nonlinearity term, $\Delta N_{i} / d_{i}$, is also negative for some species and positive for others, but because $V\left(C^{\{-i\}}\right)$ may depend on the identity of the invader, the sum of this term over species need not be zero, and indeed may be positive. As we shall see below, this fact makes it possible for $\Delta N_{i} / d_{i}$ to contribute to coexistence. Moreover, in general the storage effect term will also vary with the species. For example, in the case of a symmetric variance and covariance structure for environmental responses, but different adult death rates, the storage effect becomes

$\frac{\Delta I}{d_{i}}=\sigma^{2}\left\{\frac{(1-\rho)\left(1-\tilde{d}_{s}^{s \neq i\}}\right)}{n-1}+\rho\left(d_{i}-\bar{d}_{s}^{\{s \neq i\}}\right)\right\} B$,

where the tilde over the first $d_{s}$ indicates the weighted average over residents defined in the appendix and the bar with superscript $\{s \neq i\}$ over the second $d_{s}$ indicates the simple average of over residents. Quantity (14) may be negative for some species but averages to a positive value over each species in the community considered as an invader, $i$, provided some species live longer than one period of recruitment, viz., have $d_{i}<1$. To understand what these scenarios mean for species coexistence, we consider next the concept of community average measures of coexistence mechanisms.

\subsection{Community average measures of coexistence mechanisms}

Expression (13) for $\bar{r}_{i} / d_{i}$ has the special form

$\frac{\bar{r}_{i}}{d_{i}}=\xi_{i}+A$,

where $\xi_{i}$ and $A$ are capable of being varied independently, with the $\xi_{i}$ summing to zero over species. Note that in (13), $B$ may depend on the average of the $\mu$ values 
for the species, but not on their differences, which yield the $\xi_{i}$. Whenever long-term low-density growth rates can be put in this form with $A$ positive, then $A$ is a erm that stabilizes coexistence in the sense that without it coexistence would be impossible but in its presence the long-term low-density growth rates of all species are positive for some sets of $\xi$ values (Chesson, 2000b).

The quantities $\xi_{i}$ qualify as relative fitness measures because they quantify average relative performance of the different species in the system. The magnitude of $A$ determines the maximum average fitness disadvantage (maximum $-\xi_{i}$ ) compatible with coexistence, and thus can be regarded as a measure of the strength of the mechanism. In the particular case represented by Eq. (13), $A$ is the common contribution of the storage effect to $\bar{r}_{i} / d_{i}$ for all species in the community. In general, it makes sense that a measure of the strength of a coexistence mechanism should be a community property rather than a property of an individual species. The fact that $A$ emerges as the average over species of some scaling of $\bar{r}_{i}$ suggests that a community value for coexistence stabilizing mechanisms might be available more generally, not necessarily just as a special of feature of Eq. (13).

To see how to generalize the results of Eq. (13), we can average the individual storage-effect values given by expression (14) for the case of unequal adult death rates, but symmetric variance and covariance structure, to obtain

$$
\overline{\left(\frac{\Delta I}{d}\right)}=\sigma^{2}\left\{\frac{(1-\rho)(1-\overline{\tilde{d}})}{n-1}\right\} B,
$$

where $\overline{\tilde{d}}$ is the average of $\tilde{d}_{i}$ over each species $i$. Quantity (16) is positive, and individual deviations from this value,

$\delta I_{i}=\frac{\Delta I_{i}}{d_{i}}-\overline{\left(\frac{\Delta I}{d}\right)}$,

sum to zero, and can be a considered to be fitness differences that, like $\mu$ differences, contribute to the $\xi_{i}$. However, note that when the $d$ 's differ between species, $\Delta N_{i} / d_{i}$, will be nonzero too, and must be taken account of in $\bar{r}_{i} / d_{i}$. We can define a community average value of $\Delta N_{i} / d_{i}$, by simply averaging $\Delta N_{i} / d_{i}$ over the species $i$, which here yields

$\overline{\left(\frac{\Delta N}{d}\right)}=\frac{n}{2(n-1)} \operatorname{Cov}_{i}\left(d_{i}, V\left(C^{\{-i\}}\right)\right)$,

where the covariance is the simple covariance of $d_{i}$ and $V\left(C^{\{-i\}}\right)$ over species. Then individual relative nonlinearity deviations are

$\delta N_{i}=\frac{\Delta N_{i}}{d_{i}}-\overline{\left(\frac{\Delta N}{d}\right)}$.
With these definitions, the relative fitness measure $\xi_{i}$ is appropriately defined as

$\xi_{i}=\delta \mu_{i}+\delta V_{i}+\delta N_{i}+\delta I_{i}$,

which sums to zero as before. The stabilizing term, $A$, becomes the difference between the community average storage effect and community average relative nonlinearity of competition:

$A=\overline{\left(\frac{\Delta I}{d}\right)}-\overline{\left(\frac{\Delta N}{d}\right)}$,

because $\Delta I_{i}$ has positive sign and $\Delta N_{i}$ has a negative sign in formula (11) for $\bar{r}_{i}$. Note that if the $d$ 's are similar between species, or $V\left(C^{\{-i\}}\right)$ does not vary with $i$, then community average relative nonlinearity will be zero and relative nonlinearity in that case simply contributes to average fitness differences between species, not stability. Thus, in that case $A$ just reflects the storage effect.

If $A$ is positive, the joint action of the storage effect and relative nonlinearity of coexistence promotes species coexistence in the sense that there is then a nonempty set of $\xi_{1}, \xi_{2}, \ldots, \xi_{n}$ values for which all the species have positive growth at low density and hence coexist. This coexistence region is simply the set of all vectors $\xi=\left(\xi_{1}, \xi_{2}, \ldots, \xi_{n}\right)$ with each component greater than $-A$, and all components summing to zero. This region is a bounded $n-1$ dimensional set whose size is an increasing function of $A$. To obtain this conclusion, however, we must suppose that the $\xi$ values can be varied without changing the value of $A$. The ability to vary the $\delta \mu_{i}$ without greatly affecting the other terms of $\xi_{i}$, or $A$, attested to by results in Chesson (1994), indicates that this assumption is reasonably applicable to models of recruitment variation as defined here. In general, however, one must be concerned that the models in question are sufficiently flexible that the $\xi_{i}$ and $A$ values can be varied independently. Note that symmetry assumptions on variances and covariances required for the particular form (16) for the community average storage effect do not affect these conclusions and are not required for any conclusions below, except where specifically mentioned.

\section{Relationships of mechanisms to recruitment variation}

\subsection{Recruitment variation in residents compared with invaders}

The results above for an individual species show that the long-term low-density growth rate can be expressed as

$\bar{r}_{i} / d_{i}=\delta \mu_{i}+\delta V_{i}-\Delta N_{i} / d_{i}+\Delta I_{i} / d_{i}$, 
which expands to yield

$$
\begin{aligned}
\bar{r}_{i} / d_{i}= & \delta \mu_{i}+\delta V_{i}+\frac{1}{2}\left\{\left(1-d_{i}\right)-\overline{\left(1-d_{s}\right)}\right. \\
& \{s \neq i\} \\
& -\left(1-d_{i}\right) \chi_{i}^{\{-i\}}+\overline{\left(1-d_{s}\right) \chi_{s}^{\{-i\}}}\{s \neq i\}
\end{aligned}
$$

Using expression (8), the terms above rearrange to demonstrate the effects of recruitment variation:

$\bar{r}_{i} / d_{i}=\delta \mu_{i}+\frac{1}{2}\left\{\left(1-d_{i}\right) V\left(\ln R_{i}\right)-{\overline{\left(1-d_{s}\right) V\left(\ln R_{s}\right)}}^{\{s \neq i\}}\right\}$.

Thus, we see that recruitment variation makes a positive contribution to the persistence of an individual species when the species' recruitment variation as an invader, multiplied by its adult survival rate, is greater the average of this same quantity for resident species. When adult death rates are small, so that the $\left(1-d_{j}\right)$ can be satisfactorily approximated by 1 , and the variances of $V\left(E_{j}\right)$ of the different species are the same, the storage effect is very simply related to the difference in recruitment variation, viz.,

$\Delta I_{i} / d_{i}=\frac{1}{2}(1-d)\left\{V\left(\ln R_{i}\right)-{\overline{V\left(\ln R_{s}\right)}}^{\{s \neq i\}}\right\}$.

Thus, for a positive storage effect for species $i$, the average of resident recruitment variation has to be greater than the invader recruitment variation. In other cases, the relationship is more complicated. However, the situation is greatly simplified by making use of the development above on community average mechanisms.

Averaging expression (24) over species gives

$$
\begin{aligned}
& {\overline{\bar{r}_{i} / d_{i}}}^{\{i\}}=\frac{1}{2}\left\{{\overline{\left(1-d_{i}\right) V\left(\ln R_{i}^{\{-i\}}\right.}}^{\{i\}}\right. \text {, } \\
& \left.-{\overline{\left(1-d_{s}\right) V\left(\ln R_{s}^{\{-i\}}\right)^{\{s \neq i\}}}}^{\{i\}}\right\},
\end{aligned}
$$

where $\{-i\}$ has been added as a superscript to the recruitment rates to indicate that these are calculated for species $i$ as an invader, and the superscript $\{i\}$ on an overbar means that the average is over $i$. The results above on community-level measures of mechanisms yield

$\overline{\bar{r}}_{i} / d_{i}{ }^{\{i\}}={\overline{\Delta I_{i} / d_{i}}}^{\{i\}}-{\overline{\Delta N_{i} / d_{i}}}^{\{i\}}$.

Thus, we see that

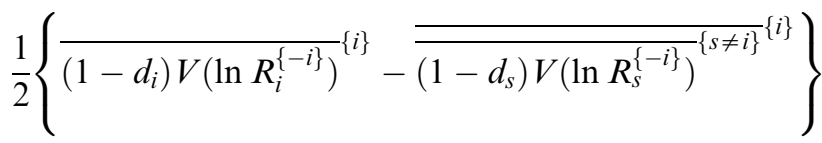

$$
\begin{aligned}
& ={\overline{\Delta I_{i} / d_{i}}}^{\{i\}}-{\overline{\Delta N_{i} / d_{i}}}^{\{i\}},
\end{aligned}
$$

i.e. average recruitment variation, weighted by the adult survival rate, comparing residents and invaders, is equal to the difference between the community-level storage effect, and community-level relative nonlinearity of competition. In particular, we see that recruitment variation has a coexistence promoting effect if average invader recruitment variation, multiplied by adult survival, is greater than average resident recruitment variation mulitplied by adult survival.

As before, when adult death rates are small, i.e. for long-lived species, the recruitment variance comparison does not need to be weighted by adult survival rates. However, in that case also, the individual and community-level relative nonlinearities should be approximately zero, and so we see in that case that the resident-invader comparison of recruitment rate variation yields the community-level storage effect. Thus, the community-level storage effect can be assessed using recruitment variation differences. This comparison would also be correct if the $d$ 's were nearly the same for different species, even if not small. In the case where the $d$ 's are not small and differ appreciably one from the other, then variance comparisons must be weighted an survival rates. Morever, in this case, coexistence potentially stems from the combined action of the storage effect and relative nonlinearity of competition (but not from other mechanisms), and the weighted recruitment comparison assesses the combined action of these two mechanisms.

As the magnitude of $d$ figures importantly in the meaning of the measures above and in the nature of the mechanisms, it is important to consider just what it means. It is defined as the fraction of adults dying in one unit of time. The units of time therefore cannot be arbitrary. As the model is defined, a unit of time allows an individual to be born and to mature as an adult. All competition that affects its survival is assumed to take place in this one interval of time. When it becomes an adult, i.e. after one unit of time, its survival is no longer affected by competition. Short-lived organisms may in fact be long-lived for our purposes here if the time from birth to maturity is short. For example, if individuals are born and become adults in the space of a day, but live 10 days on average, then they are long-lived organisms for our purposes. Differential equation models of recruitment variation (e.g., Kelly and Bowler, 2002) implicitly assume that the recruitment process is instantaneous. Thus, in these models the organisms are effectively of infinite longevity, and the adult death rates considered here are negligible, eliminating any role for relative nonlinearity of competition.

\subsection{Unperturbed systems}

The invader-resident comparison above is most applicable to experimental situations, where perturbations to the systems set it up. In contrast, we now enquire about situations where coexisting species differ in their long-run mean population densities. In this case, we assume that recruitment fluctuations have been 
observed over time in a system that has not been subject to experimental perturbation or to selection of specific times when a particular species is at unusually low density. Do differences in variance of recruitment fluctuations reflect the action of the storage effect, or more generally, a combination of the storage effect and relative nonlinearity of competition? To answer this question, we assume that the species are at a joint stationary distribution, and exhibit steady fluctuations over time. The long-term per capita growth rates of all species therefore are zero, and using the general theory of dynamics in a variable environment (Chesson, 1994), applied above, we can conclude that

$$
\begin{aligned}
0= & \mu_{s}+\frac{1}{2}\left(1-d_{s}\right) \sigma_{s}^{2}-E[C] \\
& +\frac{1}{2}\left(1-d_{s}\right) V(C)-\left(1-d_{s}\right) \chi_{s} .
\end{aligned}
$$

The unknown value $E[C]$ can be eliminated by subtracting off the mean of expression (29) over all species to obtain

$$
\begin{aligned}
0= & \mu_{s}-\bar{\mu}+\frac{1}{2}\left[\left(1-d_{s}\right) \sigma_{s}^{2}-\overline{(1-d) \sigma^{2}}\right]-\frac{1}{2}\left(d_{s}-\bar{d}\right) V(C) \\
& -\left[\left(1-d_{s}\right) \chi_{s}-\overline{(1-d) \chi}\right]
\end{aligned}
$$

where the averages are now simply taken over all species, and $C$ is for all species as residents. This expression is in a similar form to the invader growth rate (23), with terms that to correspond $\Delta N_{s} / d_{s}$ and $\Delta I_{s} / d_{s}$, but which are not actually equal to these quantities because these quantities are only defined for the invader-resident situation. However, if the mean density of species $s$ is very low, so that it has a negligible contribution to competition, the last two terms of Eq. (30) will indeed be close approximations to $\Delta N_{s} / d_{s}$ and $\Delta I_{s} / d_{s}$.

The terms in Eq. (30) combine to yield the following expression for weighted recruitment-fluctuation differences:

$0=\mu_{s}-\bar{\mu}+\frac{1}{2}\left[\left(1-d_{s}\right) V\left(\ln R_{s}\right)-\overline{(1-d) V(\ln R)}\right]$.

Thus, when comparing different species in a given community, the mean fitness value $\mu_{s}$ is negatively related to recruitment variance weighted by the adult survival rate. Although, this variance comparison does not precisely measure the coexistence mechanisms in the same way as the resident-invader comparisons above, nevertheless, the relationship that it implies between recruitment variation and mean fitnesses is an outcome of the assumption that $C$ is common to coexisting species. This assumption is only compatible with recruitment variation as the mechanism of coexistence, and so relationship (31) can be used to test recruitment variation as the coexistence mechanism within the constraints of the models considered here. We first ask if mean fitness might be positively related to mean population densities, meaning that the negative relationship between mean fitness and recruitment variance implies a negative relationship between mean densities and recruitment variation, which would be more readily observed, and is the test of the storage effect used by Kelly and Bowler (2002).

In some models, for example, the lottery model with equal adult death rates, and symmetric variances and covariances as defined in the section on coexistence conditions above, mean density, $E\left[N_{s}\right]$, is a simple increasing function of $\mu_{s}$ (available from the joint stationary distribution for the lottery model found in Hatfield and Chesson, 1997). As the adult death rates are the same, this result implies that recruitment variance decreases as mean density increases. However, in the multispecies lottery model, the mean densities can differ between species when the $\mu$ 's are the same for all species but the adult death rates differ between species (Chesson, 1984). In this case, the mean densities are proportional to the mean longevities, $1 / d_{s}$, in long-lived species. However, as (31) then implies that recruitment variance is proportional to $1 /\left(1-d_{s}\right)$, this outcome again means that mean densities and recruitment fluctuations are negatively related. The situation is not so straightforward, however, if both the $\mu$ 's and the $d$ 's differ between species, as discussed below.

For empirical investigations, it would be desirable to be able to remove as many restrictions as possible on the existence of a positive relationship between mean abundance and recruitment variance. Kelly and Bowler (2002) specifically investigated the two-species situation of the lottery model where one species has low $\sigma^{2}=$ $V(E)$ and other has high $\sigma^{2}$, with equal adult death rates. However, in the two-species lottery model with equal death rates, mean densities depend only on the $\mu$ values and the variance of the difference in the $E$ values, not their separate variances (Hatfield and Chesson, 1997). Thus, higher $E[N]$ continues to correspond to higher $\mu$ and lower $V(\ln R)$.

In general, decreasing $\mu$ of any given species, while keeping other parameters constant, must ultimately decrease $E[N]$ to arbitrarily low levels because it is by this means that a species' long-term low-density growth rate $\left(\bar{r}_{i}\right)$ can be made arbitrarily low. Thus, intraspecific density dependence will lead to negative average growth rates at arbitrarily low densities. Hence, in the limit as $\mu$ becomes small, $E[N]$ must reflect $\mu$. However, without restrictions beyond those needed to derive Eq. (31), $E[N]$ cannot be expected to be monotonically related to $\mu$ within a given set of coexisting species. For example, in the two-species lottery model with small but unequal adult death rates, it is possible to see from the formulae in Hatfield and Chesson (1989) that the species with the smaller $\mu$ can nevertheless have the larger mean population density if its adult death rate is sufficiently small relative to that of the other species. However, for small adult death rates, Eq. (31) implies that the variance difference will reflect the $\mu$ difference, unless the $\mu$ difference is small too. Thus, although within a 
community $V(\ln R)$ is negatively related to $\mu$ whenever the species have either small or equal adult death rates this relationship does not always carry over to a negative relationship within a community between $V(\ln R)$ and $E[N]$.

The difficulties of being sure that $E[N]$ reflects $\mu$ suggest that basing the comparison between variances on the comparison between $\mu$ values is the approach to take. This approach requires some means, perhaps experimental, of measuring mean $\mathrm{ln}$ recruitment rates in the absence of competition, or at fixed levels of competition. One should avoid the temptation of calculating $\mu$ differences as mean recruitment differences, an equation implied by the assumption that $C$ is common to the species in the community, because this assumption implies that coexistence stems from recruitment fluctuations. Thus, the prediction that smaller $\mu$ corresponds to larger recruitment variation would necessarily be borne out regardless of the operation of the mechanism. A means of assessing $\mu$ differences that does not invoke this assumption is therefore necessary.

\subsection{Generalization}

The general model with all species having a common value for the competitive response, $C_{j}$, makes the assumption that the species are affected similarly by competition to the extent that the change in ln survival of recruits due to competition is the same for all species at any given time. In demographic jargon, this means that the contribution of competition to the force of mortality is the same for all species. This assumption is justified by models like the lottery model where competition for space imposes uniform restrictions on all species. However, other models can be envisaged where this might not be the case. We can generalize the model by simply making the assumption that the $C_{j}$ for different species are linearly related. Thus, we have the equation

$C_{j}=a_{j} C$,

where $C$ is a common, potentially composite, factor underlying all species, and $a_{j}$ is a species-specific constant. This model then allows different species to be sensitive to different degrees to the underlying competitive factors, but in essence to respond to them in the same way. The existence of this common $C$ precludes coexistence in the absence of recruitment fluctuations, and so we remain within the class of situations where species coexistence must be explained by them, and we seek to understand how to detect this fact in nature. Eq. (32) might be generalized further by having $C_{j}$ simply be some increasing function, different for different species, of the competitive factor $C$; but unless such functions are strongly relatively nonlinear, they are unlikely to significantly affect the results here, which are valid whenever these functions can be adequately approximated linearly in terms of underlying factors. A more general linear form than (32) would be $C_{j}=$ $b_{j}+a_{j} C$, but the constant $b_{j}$ can simply be absorbed into $E_{j}$ without affecting anything in the development here. The parameter $a_{j}$ is termed sensitivity to competition in Chesson and Huntly (1997), and we shall call it that here.

Several changes occur with the introduction of the constant $a_{j}$, following the techniques of Chesson (1994). First, the growth rates take the form (15) when $\bar{r}_{i}$ is divided by $d_{i} a_{i}$ rather by $d_{i}$ alone. Second, relative nonlinearity of competition involves the comparison between species of the values of $\left(1-d_{j}\right) a_{j}$ rather than simply the surival rates $\left(1-d_{j}\right)$. Finally, covariance between environment and competition $\chi_{j}^{\{-i\}}$ is defined in terms of $C_{j}$ as $\chi_{j}^{\{-i\}}=\operatorname{Cov}\left(E_{j}, C_{j}^{\{-i\}}\right)=a_{j} \operatorname{Cov}\left(E_{j}, C^{\{-i\}}\right)$. Having made these adjustments, the critical expressions (23) and (24) become

$$
\begin{aligned}
\bar{r}_{i} / a_{i} d_{i}= & \delta \mu_{i}+\delta V_{i}+\frac{1}{2}\left\{a_{i}\left(1-d_{i}\right)-{\overline{a_{s}\left(1-d_{s}\right)}}^{\{s \neq i\}}\right\} \\
& \times V\left(C^{\{-i\}}\right)-\frac{1-d_{i}}{a_{i}} \chi_{i}^{\{-i\}}+\frac{{\overline{1-d_{s}}}_{a_{s}} \chi_{s}^{\{-i\}}}{\{s \neq i\}}
\end{aligned}
$$

and

$$
\begin{aligned}
\bar{r}_{i} / a_{i} d_{i}= & \delta \mu_{i}+\frac{1}{2}\left\{\frac{1-d_{i}}{a_{i}} V\left(\ln R_{i}\right)\right. \\
& \left.-\frac{{ }^{1-d_{s}}}{a_{s}} V\left(\ln R_{s}\right)^{s \neq i\}}\right\},
\end{aligned}
$$

where $\mu_{i}$ is defined as $\left(E\left[E_{i}\right]-\ln d_{i}\right) / a_{i}, \delta \mu_{i}=\mu_{i}-\bar{\mu}_{s}\{s \neq i\}$, and $\delta V_{i}=\frac{1}{2}\left\{\left(1-d_{i}\right) \sigma_{i}^{2} / a_{i}-\overline{\left(1-d_{s}\right) \sigma_{s}^{2} / a_{s}}\{s \neq i\}\right\}$. Of most importance is the relationship between average invaderresident recruitment variation differences and community average measures of mechanisms, which here reduces to

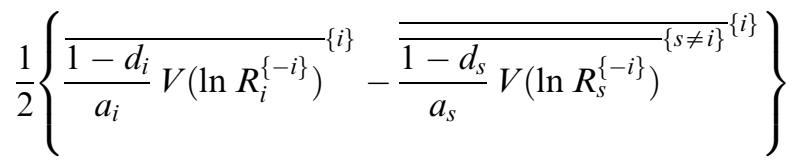

$$
\begin{aligned}
& ={\frac{\overline{\Delta I}_{i}}{a_{i} d_{i}}}^{\{i\}}-{\overline{\overline{\Delta N}_{i}}}_{a_{i} d_{i}}^{\{i\}} .
\end{aligned}
$$

The right-hand side of this equation is the difference between the community-level storage effect, and the community-level relative nonlinearity. The left-hand side is again an average-weighted comparison of recruitment variation between invaders and residents. The weights, however, do not necessarily become unimportant in long-lived organisms. The comparison remains irreducibly dependent on the relative sensitivities to the common competitive factors of juvenile mortality rates. Similar dependence occurs for the comparison of recruitment variation for long-term low-density growth rates of individual species 
(Eq. (34)) and the counterpart to Eq. (31) for the relationship between $\mu_{s}$ and recruitment variation for unperturbed systems. Thus, some appreciation of the relative sensitivities of juvenile mortality rates to common competitive factors is necessary to make effective use of recruitment variation comparisons to test species coexistence mechanisms.

\section{Discussion}

The results here suggest that coexistence mechanisms relying on recruitment fluctuations should be detectable by comparing the variances of $\ln$ recruitment ("recruitment variance") of the different species in a community. The outcomes of these comparisons can be regarded as tests of the hypothesis that recruitment fluctuations are responsible for coexistence. They are predictions of the general model given here in which recruitment fluctuations are the only possible coexistence mechanism. They are not predictions of alternative models in which coexistence results from factors such as resource partitioning or frequency-dependent predation. However, for resident-invader comparisons, predictions applicable to the sole action of recruitment fluctuations may also apply to a combination recruitment fluctuations and other mechanisms, as implied by formulae for such situations in Chesson (1994). Thus, passing these tests, while implicating recruitment fluctuations, does not rule out the presence of other mechanisms.

The simplicity of comparisons assessing the role of recruitment fluctuations in species coexistence varies with the circumstances. The clearest comparisons are possible for invaders in relation to residents, as defined in an invasion analysis of species coexistence where individual species are kept at low density, and their recruitment rates are compared with those in a resident community. Simple predictions are then available for species that have similar sensitivity to competition (measured by the parameter $a$ above) and are either long-lived or have similar longevities. In these cases, average recruitment variance is predicted unambiguously to be higher for invaders than residents. Moreover, the difference between invader and resident recruitment variance is proportional to the standard quantitative measure of the storage effect for an individual invader, and the average invader variance compared with average resident variance is proportional to the community average storage effect. If the species are not long-lived and have different adult longevities, the situation is a little more complicated, because then the relevant comparison is between recruitment variances multiplied by adult survival rates. However, any appreciable knowledge of recruitment variation is likely to be accompanied by a knowledge of adult longevity, and this requirement is no serious difficulty in practice.

With differences in adult longevity for species that are not long-lived, the storage effect is not necessarily the sole mechanism of coexistence arising from recruitment fluctuations. The mechanism termed relative nonlinearity of competition may also have a role. Average differences between survival-weighted recruitment variances for invaders and residents then measure the joint effect of the storage effect and relative nonlinearity of competition. More serious complications occur when species have different sensitivities to competition. Then sensitivity to competition has to be factored into the comparison between recruitment variances. Space competition, as exemplified by the lottery model, implies that species should not differ in sensitivity to competition. Similar scenarios in which gaining a certain amount of resource is necessary for recruitment (like a unit of space in the lottery model), also argue for equal sensitivities to competition, because these models can be formulated as variations on the lottery model preserving equal sensitivity even when different species require different amounts of resource for recruitment. If other models are thought necessary in particular circumstances, then experimental measurement of sensitivity to competition may be necessary to apply the methods here.

The invader-resident comparison is one form of comparison between high- and low-density species, but is not necessarily the most natural such comparison. The case where species' recruitment fluctuations are simply observed with no species constrained to low density, however, still leads to clear predictions. These predictions most straightforwardly involve not the actual mean densities of the species, but quantities related to them, viz., the mean fitness components denoted by $\mu$ above. However, to use this approach, means of assessing these mean fitness components need to be developed along with methods of assessing sensitivity to competition. In general, such methods may be difficult to develop and could severely limit the ability to assess the role of recruitment variation in coexistence outside resident-invader comparisons.

Invader-resident comparisons, if they can be done, provide the clearest assessments of coexistence mechanisms because they are capable of measuring their full magnitude. They remain applicable even if species have similar average fitness components and similar mean densities as residents. The main difficulty is setting up these comparisons. The ideal situation is to experimentally perturb a species to low density and maintain it there artificially, while allowing the rest of the species to approach the stationary distribution that they have in the absence of this particular species. Such a situation is most easily imagined in the laboratory with very small organisms whose populations turn over quickly. 
Warner and Chesson (1985) suggest procedures for nonexperimental investigation of recruitment variation in species approximating the invader-resident scenario, and give some examples using small marine invertebrates. Fundamentally, stochastic fluctuations will mean that species densities may range widely, and some observations of recruitment of a species while at low density might be regarded as equivalent to its recruitment as an invader while recruitment of other species at those times is equivalent to their recruitment as residents in the absence of the species assuming the invader role. Ideally, residents should be at their stationary distribution whenever such observations are made, and so a key question is how quickly this distribution is approached. In the two-species lottery model, the resident reaches its stationary distribution in one recruitment period, and so all observations for a species at low density can be regarded as measuring that species and its competitor in an invader-resident state. In other cases, a given species may have to be at low density for several to many recruitment periods before this assumption is satisfactory. Care would also have to be taken in case environmental fluctuations affecting recruitment are autocorrelated on the timescales considered, for then unfavorable conditions causing a particular species to be at low density might tend to persist biasing observations of recruitment variation toward unfavorable environmental conditions. Although these challenges of approximating an invader-resident scenario may seem daunting, with small organisms and fast generation times, it seems possible. Moreover, steadily improving methods of historical reconstruction of community dynamics (Davis et al., 1994; Jackson et al., 2001) open the possibility of obtaining very long time series of recruitment fluctuations for some communities making these methods feasible and powerful.

In the process of developing relationships between recruitment fluctuations and the fundamental coexistence mechanisms, we also considered and developed the new concept of community average measures of mechanisms. The long-term low-density growth rate $\left(\bar{r}_{i}\right)$ is then partitioned into components in terms of these community average measures, and components expressing average fitness differences, which sum to zero over species. This technique, which here clarified the relationship between recruitment fluctuations and the fundamental mechanisms, also furthers understanding of the two fundamental kinds of coexistence mechanism discussed in Chesson (2000b), viz., stabilizing and equalizing mechanisms. Stabilizing mechanisms are those allowing species to have positive invader growth rates, in spite of average fitness differences. Equalizing mechanisms are means by which average fitness differences are reduced. From the work here, we see that the storage effect and relative nonlinearity of competition can have both stabilizing and equalizing (or unequalizing) components. The community average measures of the storage effect and relative nonlinearity of competition summarize their overall contributions to stabilizing coexistence. Individual species deviations from these community average values then contribute to average fitness differences between species and may trade off against other average fitness components arising in other ways. Such procedures are generally applicable to species coexistence mechanisms and potentially provide a powerful new tool for analyzing species coexistence.

\section{Acknowledgments}

I am grateful for comments on this manuscript by Michael Bowler, Colleen Kelly, Charlotte Lee, Robin Snyder and an anonymous reviewer. This work was supported by NSF grant OCE-9911386.

\section{Appendix}

The analysis of the lottery model in Chesson (1994) is partly applicable to the general recruitment variation model considered here because the lottery model also has the same function $g_{j}$, (Eq. (10)) as the general recruitment variation model. Moreover, the comparison of the small-effects approximation for the lottery model with numerical results (Hatfield and Chesson, 1989) suggests that this approximation is broadly satisfactory for the general recruitment variation model. Most of the results here depend on this approximation.

To define the first term, $\bar{r}_{i}^{\prime}$, we first choose a reference value $C^{*}$ of competition, and define

$\mathscr{E}_{i}=\ln \left(s_{i}+e^{E_{i}-C^{*}}\right)$,

which can be thought of as an average measure of fitness of species $i$ in the system at the reference value of competition, $C^{*}$. Then, $\bar{r}_{i}$ becomes

$\bar{r}_{i}^{\prime}=E\left[\mathscr{E}_{i}\right]-{\overline{E\left[\mathscr{E}_{S}\right]}}^{\{s \neq i\}}$,

where the superscript $\{s \neq i\}$ on the bar in (A.2) means that the average is over all the resident $E\left[\mathscr{E}_{s}\right]$ values. The actual value of (A.2) depends only weakly on the reference value $C^{*}$. A suitable choice for $C^{*}$ is $\frac{1}{n} \sum_{i} E\left[C^{\{-i\}}\right]$, the average of the expected competition over each of the $n$ species considered in turn as an invader. Having made this choice, the $E\left[\mathscr{E}_{j}\right]$ are uniquely defined. Moreover, expression (A.2) cannot vary by more than $o\left(\sigma^{2}\right)$ where $\sigma^{2}$ is a small parameter expressing the common order of magnitude of the variances, $V\left(E_{j}\right)$, provided we make the standard assumptions of small-effects approximations (Chesson, 1994) and assume that $C^{*}$ varies from $\frac{1}{n} \sum_{i} E\left[C^{\{-i\}}\right]$ by no more than $O\left(\sigma^{2}\right)$. The value of $E\left[\mathscr{E}_{j}\right]$ reflects both the 
mean and the variance of $E_{j}$. Specifically

$E\left[\mathscr{E}_{j}\right] / d_{j}=\mu_{j}+\frac{1}{2}\left(1-d_{j}\right) \sigma_{j}^{2}-C^{*}$.

Thus

$$
\begin{aligned}
\bar{r}_{i}^{\prime} / d_{i} & =\mu_{i}-{\overline{\mu_{s}}}_{s}\{s \neq i\}+\frac{1}{2}\left(1-d_{i}\right) \sigma_{i}^{2}-\overline{\frac{1}{2}\left(1-d_{s}\right) \sigma_{s}^{2}}\{s \neq i\} \\
& =\delta \mu_{i}+\delta V_{i},
\end{aligned}
$$

as given in Table 1 .

The other rows of Table 1 are immediate from the analysis of the lottery model in Chesson (1994). Note, however, that the covariances are in different units ("standardized units") than those in Table 1. However, covariances in terms of standardized and original units are interconvertible as explained in Chesson (1989). In Chesson (1994) the notation $\chi_{j j}^{-i}$ is used for covariance between environment and competition, which is simplified here to $\chi_{j}^{\{-i\}}$ because the double subscript serves no purpose here. The general expressions in Table 1 do not require the symmetric variance assumption used in the text for the special storage-effect formulae (12) and (14).

To obtain expressions (12) and (14), we introduce a general model of competition where

$C=f\left(\sum_{l} a_{l}(\mathbf{N}) e^{E_{l}} N_{l}\right)$.

Here $f$ is an increasing function, and the competitive effects $a_{l}(\mathbf{N})$ of individual juveniles of species $l$ are now functions of the vector $\mathbf{N}=\left(N_{1}, N_{2}, \ldots, N_{n}\right)$ of adult densities of the species in the system. This formula generalizes both competition expressions (6) and (7) in the text. Applying the symmetric variance assumption of the text and (Chesson, 1994, expression (79)), it follows that

$\Delta I_{i}=d_{i} \sigma^{2}\left\{\frac{(1-\rho)\left(1-\tilde{d}_{s}^{\{s \neq i\}}\right)}{n-1}+\rho\left(d_{i}-\bar{d}_{s}^{\{s \neq i\}}\right)\right\} B$,

where $B$ and $\tilde{d}_{s}^{\{s \neq i\}}$ are defined in terms of $\alpha_{s} a_{s}=$ $E\left[\partial C^{\{-i\}} / \partial E_{s}\right]$ as

$\tilde{d}_{s}^{\{s \neq i\}}=\sum_{s \neq i} \alpha_{s} a_{s} d_{s} / \sum_{s \neq i} \alpha_{s} a_{s}$

and

$$
\begin{aligned}
B=\sum_{s \neq i} \alpha_{s} a_{s} & =E\left[f^{\prime}\left(\sum_{s \neq i} a_{s}(\mathbf{N}) e^{E_{s}} N_{s}\right) \sum_{s \neq i} a_{s}(\mathbf{N}) e^{E_{s}} N_{s}\right] \\
& =E\left[f^{\prime}\left(f^{-1}\left(C^{\{-i\}}\right)\right) f^{-1}\left(C^{\{-i\}}\right)\right] .
\end{aligned}
$$

Now if $f^{\prime} \circ f^{-1} \times f^{-1}$ is a smooth function, results in Chesson (1994, Appendix II) show that value (A.8) is within $O\left(\sigma^{2}\right)$ of $f^{\prime}\left(f^{-1}\left(C^{*}\right)\right) f^{-1}\left(C^{*}\right)$. It can thus be considered a constant as the parameters of the model are varied. For the lottery model, the formula $f^{\prime}\left(f^{-1}\left(C^{*}\right)\right) f^{-1}\left(C^{*}\right)$ for $B$ gives the value 1 , and for the model given by Eq. (6) it is $C^{*}$. Note that expression (12) of the text is just the special case of (A.6) with $d_{j}$ the same for all species. Expression (14) is simply (A.6).

\section{References}

Abrams, P.A., 1984. Recruitment, lotteries, and coexistence in coral reef fish. Am. Nat. 123, 44-55.

Abrams, P., Holt, R., 2002. The impact of consumer-resource cycles on the coexistence of competing consumers. Theor. Popul. Biol. 62, 281-295.

Baskin, C.C., Chesson, P., Baskin, J.M., 1993. Annual seed dormancy cycles in two desert winter annuals. J. Ecol. 81, 551-556.

Chesson, P.L., 1982. The stabilizing effect of a random environment J. Math. Biol. 15, 1-36.

Chesson, P.L., 1984. The storage effect in stochastic population models. Lect. Notes Biomath. 54, 76-89.

Caceres, C.E., 1997. Temporal variation, dormancy, and coexistence: a field test of the storage effect. Proc. Natl. Acad. Sci. USA 94, 9171-9175.

Chesson, P.L., 1989. A general model of the role of environmental variability in communities of competing species. Am. Math. Soc.: Lect. Math. Life Sci. 20, 97-123.

Chesson, P.L., 1990. Geometry, heterogeneity and competition in variable environments. Philos. Trans. R. Soc. London, Ser. B 330, 165-173.

Chesson, P., 1994. Multispecies competition in variable environments. Theor. Popul. Biol. 45, 227-276.

Chesson, P., 2000a. General theory of competitive coexistence in spatially varying environments. Theor. Popul. Biol. 58, 211-237.

Chesson, P., 2000b. Mechanisms of maintenance of species diversity. Annu. Rev. Ecol. Syst. 31, 343-366.

Chesson, P., Huntly, N., 1989. Short-term instabilities and long-term community dynamics. Trends Ecol. Evol. 4, 293-298.

Chesson, P., Huntly, N., 1997. The roles of harsh and fluctuating conditions in the dynamics of ecological communities. Am. Nat. 150, 519-553.

Chesson, P.L., Warner, R.R., 1981. Environmental variability promotes coexistence in lottery competitive systems. Am. Nat. 117, 923-943.

Chesson, P., Pacala, S., Neuhauser, C., 2001. Environmental niches and ecosystem functioning. In: Kinzig, A., Pacala, S., Tilman, D. (Eds.), The Functional Consequences of Biodiversity. Princeton University Press, Princeton, NJ, pp. 213-245.

Davis, M.B., Sugita, S., Calcote, R.R., Ferrari, J.B., Frelich, L.E., 1994. Historical development of alternate communities in a Hemlock-hardwood forest in Northern Michigan, USA. In: Edwards, P.J., May, R.M., Webb, N.R. (Eds.), Large-Scale Ecology and Conservation Biology. Blackwell Scientific Publications, London, pp. 2.

Dewi, S., Chesson, P., 2003. The age-structured lottery model. Theor. Popul. Biol. 64, 331-343.

Dixon, P.A., Milicich, M.J., Sugihara, G., 1999. Episodic fluctuations in larval supply. Science 283, 1528-1530.

Doherty, P., Fowler, A., 1994. Demographic consequences of variable recruitment to coral reef fish populations: a congeneric comparison of two damselfishes. Bull. Mar. Sci. 54, 297-313.

Ellner, S., 1989. Convergence to stationary distributions in two-species stochastic competition models. J. Math. Biol. 27, 451-462.

Grace, J.B., 1995. On the measurement of plant competition intensity. Ecology 76, 305-308.

Hatfield, J., Chesson, P.L., 1989. Diffusion approximation and stationary distribution for the lottery competition model. Theor. Popul. Biol. 36, 251-266.

Hatfield, J., Chesson, P., 1997. The multispecies lottery competition model: a diffusion analysis. In: Tuljapurkar, S., Caswell, H.E. 
(Eds.), Structured Population Models in Marine, Freshwater, and Terrestrial Systems. Chapman \& Hall, New York, pp. 615-622.

Hubbell, S.P., 2001. The Unified Neutral Theory of Biodiversity and Biogeography. Princeton University Press, Princeton, NJ.

Huisman, J., Weissing, F.J., 2002. Oscillations and chaos generated by competition for interactively essential resources. Ecol. Res. 17, 175-181.

Jackson, J.B.C., Kirby, M.X., Berger, W.H., Bjorndal, K.A., Botsford, L.W., Bourque, B.J., Bradbury, R.H., Cooke, R., Erlandson, J., Estes, J.A., Hughes, T.P., Kidwell, S., Lange, C.B., Lenihan, H.S., Pandolfi, J.M., Peterson, C.H., Steneck, R.S., Tegner, M.J., Warner, R.R., 2001. Historical overfishing and the recent collapse of coastal ecosystems. Science 293, 629-637.

Kelly, C.K., Bowler, M.G., 2002. Coexistence and relative abundance in forest trees. Nature 417, 437-440.
Pake, C.E., Venable, D.L., 1995. Is coexistence of Sonoran desert annuals mediated by temporal variability in reproductive success? Ecology 76, 246-261.

Pake, C.E., Venable, D.L., 1996. Seed banks in desert annuals: implications for persistence and coexistence in variable environments. Ecology 77, 1427-1435.

Turelli, M., 1978. Does environmental variability limit niche overlap? Proc. Natl. Acad. Sci. USA 75, 5085-5089.

Turelli, M., 1981. Niche overlap and invasion of competitors in random environments I: models without demographic stochasticity. Theor. Popul. Biol. 20, 1-56.

Warner, R.R., Chesson, P.L., 1985. Coexistence mediated by recruitment fluctuations: a field guide to the storage effect. Am. Nat. 125, $769-787$. 